\title{
Clinically accessible tools for documenting the impact of orthostatic intolerance on symptoms and function in ME/CFS
}

\author{
Jihyun Lee, Pelle Wall, Chris Kimler, Lucinda Bateman and Suzanne D. Vernon* \\ The Bateman Horne Center of Excellence, Salt Lake City, UT, USA
}

Received 2 August 2019

Accepted 16 December 2019

\begin{abstract}
.
BACKGROUND: Clinical observations have indicated that hours of upright activity (HUA) reported by Myalgic Encephalomyelitis/Chronic Fatigue Syndrome (ME/CFS) patients correlated with orthostatic symptoms and impaired physical function. This study examined the relationship between HUA and orthostatic intolerance (OI).

METHODS: Twenty-five female ME/CFS subjects and 25 age and race matched female healthy controls (HCs) were enrolled. Subjects reported HUA (defined as hours per day spent with feet on the floor) and completed questionnaires to assess the impact of OI on daily activities and symptoms. ME/CFS patients were categorized into those with $<5$ HUA and $\geq 5$ HUA and analyzed by employment status. Data analysis used one-way ANOVA.

RESULTS: ME/CFS patients had fewer HUA, worse symptoms and greater interference with daily activities due to OI than HCs. The $<5$ HUA ME/CFS subjects had more severe OI related symptoms than $\geq 5$ HUA ME/CFS subjects even though OI interfered with daily activities similarly. Only $33 \%$ of ME/CFS subjects were employed and all were $\geq 5$ HUA ME/CFS subjects with an average HUA of 8 .

CONCLUSIONS: ME/CFS subjects experienced more frequent and severe OI symptoms, higher interference with daily activities, and reduced ability to work than HCs. Reported HUA and assessment of OI using standardized instruments may be useful clinical tools for physicians in the diagnosis, treatment and management of ME/CFS patients.
\end{abstract}

Keywords: ME/CFS, hours of upright activity, orthostatic intolerance, impaired physical function

\section{Introduction}

Myalgic Encephalomyelitis/Chronic Fatigue Syndrome (ME/CFS) affects as many as 2.5 million people in the United States. ME/CFS is a debilitating disease with a significant unmet medical need that creates enormous burdens for patients, caregivers, the healthcare system and society [1]. Because fatigue is one of the most common complaints in primary

\footnotetext{
*Address for correspondence: Suzanne D. Vernon PhD, The Bateman Horne Center of Excellence, 24 South 1100 East, Suite 205, Salt Lake City, UT 84102, USA. Tel.: +1 801839 6229; Fax: +1 801364 3528; E-mail: sdvernon@batemanhornecenter.org.
}

care, as part of the differential diagnosis of ME/CFS it is critical to describe the severity and impact of fatigue on physical function. The fatigue observed in ME/CFS patients is typically more severe, more devastating and of longer duration than the fatigue level observed in other disorders characterized by fatigue. There is sufficient evidence that ME/CFS, where by the definition of fatigue persists for more than 6 months, is a serious illness that results in significant degradation or destruction of the ability to retain pre-illness levels of daily functioning (occupation, education, personal and social activity) [1]. The fatigue of ME/CFS is described by patients 
as "exhaustion, weakness, a lack of energy, feeling drained, an inability to stand for even a few minutes, an inability to walk even a few blocks without exhaustion, and an inability to sustain an activity for any significant length of time" [2]. This type of fatigue can make it hard to sustain even minor daily activities such as changing clothes, toileting or even speaking [2]. Therefore, not only the severity of fatigue as a symptom, but also the impact of fatigue on function should be assessed when making a diagnosis of ME/CFS.

Orthostatic stress worsens fatigue and cognitive function in many patients with ME/CFS [3, 4]. Orthostatic intolerance (OI) is defined as the development of symptoms upon assuming and maintaining upright postures that are alleviated by recumbency $[5,6]$. "Symptoms of orthostatic intolerance are those caused primarily by 1) cerebral under-perfusion (such as lightheadedness, near-syncope or syncope, impaired concentration, headaches, and dimming or blurring of vision), or 2) sympathetic nervous system activation (such as forceful beating of the heart, palpitations, tremulousness, and chest pain), and 3) other common signs and symptoms of orthostatic intolerance such as fatigue, a feeling of weakness, intolerance to low-impact exercise, nausea, abdominal pain, facial pallor, nervousness, and shortness of breath." [6]. Symptoms of OI are exacerbated by prolonged periods of upright posture and various daily experiences such as exercise, warm environments or large meals. Circumstances in daily life that can easily exacerbate OI include standing in line, grocery shopping, hot weather, overheated rooms, hot showers or baths and even sitting to eat a meal.

Clinicians should document patients' symptoms and function in order to determine the impact of illness on the performance of daily activities and occupational responsibilities. A medical record that documents impaired function caused by ME/CFS is crucial for patients who need to claim Social Security Disability. A major barrier to disability support relates to the absence of ME/CFS in the Social Security List of Impairments, although there is a Social Security Regulation (SSR-14-1) that explains how impairments of ME/CFS constitute a determinable condition [7]. Regardless of disability status, the ability to measure impaired function is an important aspect of diagnosis, treatment, and assessment of treatment outcomes [7].

Our clinical experience with over 1,000 ME/CFS patients has indicated that their disease severity can be gauged by hours of upright activity which we define as time with feet on the floor over a 24-hour period. Severely ill ME/CFS patients reported 0 to 4 hours with their feet on the floor while moderately ill patients reported having their feet on the floor for 5 to 8 hours. This observation led us to explore which $\mathrm{ME} / \mathrm{CFS}$ symptoms were associated with an upright activity. In this study, subjects were asked to report hours of upright activity (HUA) by estimating the hours per day spent in the following 4 positions over the past week: 1) hours upright (standing, walking, running), 2) hours sitting with feet on the floor, 3) hours reclining or sitting with feet elevated and, 4) hours lying down (includes sleeping). We also used a standardized orthostatic questionnaire to evaluate how being in upright positions affected daily activities and the severity of symptoms. We found that both assessment tools can be easily administered and are effective at documenting the impact of orthostatic intolerance on symptom severity and daily activities in ME/CFS patients.

\section{Methods}

\subsection{Study subjects}

This study was reviewed and approved by the Western Institutional Review Board (WIRB) protocol number 20170248. Participants were referred directly to the Bateman Horne Center (BHC) research department, or voluntarily presented from the BHC clinic (Salt Lake City, UT) between April 2017 and August 2017. Enrolled ME/CFS subjects were required to fulfill the International Chronic Fatigue Syndrome Study Group research criteria [8], the Canadian Clinical Criteria [9], and the IOM clinical diagnostic criteria [1]. A visit to $\mathrm{BHC}$ was required for clinical evaluation to ensure all subjects satisfied the inclusion and exclusion criteria. Therefore, homebound and bedbound ME/CFS subjects are not represented. A total of 25 women with ME/CFS and a range of moderate to severe physical and cognitive impairment were recruited to the study. In addition, 25 female healthy controls (HCs) were recruited from the Utah area using advertisements posted on social media, the Bateman Horne Center webpage or by phone contact with a volunteer pool from previous studies. HCs were matched with ME/CFS cases by age ( \pm 5 years) and ethnicity. The age range of ME/CFS and HCs was 18-65 years at the time of informed consent. Exclusion criteria for ME/CFS subjects included the presence of any active confounding diseases or events 
that explain most of the major symptoms of fatigue or place the subject at risk, based on the professional opinion of the attending physician and principal investigator. Examples are listed below:

- Organ failure (ex. Cirrhosis, cardiac failure, or chronic renal failure)

- Chronic infectious disease (ex. tuberculosis, chronic hepatitis, HIV, Lyme disease)

- Rheumatic and chronic inflammatory disease (ex. inflammatory bowel disease, rheumatoid arthritis, or chronic pancreatitis)

- Major neurological disease (ex. multiple sclerosis, stroke, traumatic brain injury, epilepsy or untreated B12 deficiency)

- Untreated or severe primary sleep disorder

- Major surgery within 6 months or minor surgery within 3 months before enrollment

- Major infection within 3 months before enrollment

- Myocardial infarction or heart failure 5 years or less before enrollment

- Morbidity obesity (BMI $>40)$

- Pregnancy, less than 3 months postpartum, or breast feeding

- Severe psychiatric illness (ex. bipolar 1 disorder, schizophrenia, anorexia nervosa)

Exclusion criteria for HCs required that subjects not meet the clinical criteria for ME/CFS nor have a prior diagnosis or history of ME/CFS. HCs also met the same exclusion criteria as ME/CFS subjects, based on the professional opinion of the attending physician and principal investigator.

\subsection{Hours of upright activity (HUA)}

HUA is defined as the amount of time spent with feet on the floor over a 24-hour period, including seated with feet on floor and standing, walking or running. Non-upright activity is defined as reclining or sitting with feet elevated and lying down (including sleeping time).

\subsection{Orthostatic Intolerance Questionnaire (OIQ)}

To measure OI we used the Orthostatic Hypotension Questionnaire (OHQ) [10], and because ME/CFS patients do not frequently have formally defined orthostatic hypotension as the presenting sign, we changed the text from "orthostatic hypotension" to "orthostatic intolerance" when we administered the questionnaire. For this reason, the OHQ is referred to as the OIQ (Orthostatic Intolerance Questionnaire) for this study. This questionnaire was developed with two subscales: the six-item Orthostatic Hypotension Symptom Assessment scale and four-item Orthostatic Hypotension Daily Activity Scale again replacing hypotension with intolerance yielding subscale acronyms of OISA and OIDAS, respectively. Each subscale is scored on a $0-10$ scale, with higher scores reflecting more severe symptoms compared to few/no symptoms at lower scores. The OISA has a total possible score of 40 and the OIDAS a total possible score of 60. Added together they constitute the OIQ with a total possible score of 100 . OISA measures 6 orthostatic intolerance symptoms that occur from being upright, including; 1) dizziness, lightheadedness, feeling faint, or feeling like you might blackout, 2) problems with vision (blurring, seeing spots, tunnel vision, etc.), 3) weakness, 4) fatigue, 5) trouble concentrating, and 6) head-neck discomfort. Subjects are asked to rate each item by checking the number on a 0 to 10 scale (None 0 - Worst Possible 10) that best represents how much the symptom has been experienced from being upright, on average, over the past week. OIDAS measures the impact of orthostatic intolerance on daily activity using 4 items; 1) standing a short time, 2) standing a long time, 3) walking a short time, and 4) walking a long time. Subjects are asked to rate each item by checking the number on a 0 to 10 scale $(0$ is no interference to 10 being complete interference) that best represents how much the symptoms of upright posture have interfered with activity, on average, over the past week. If the subjects cannot do the activity for reasons other than being upright, they are asked to check the box "Cannot do for other reasons".

\subsection{Statistical analyses}

Data analyses used STATA (IC 15.0 for windows 64-bit) statistical software. We compared demographic characteristics between the groups using descriptive statistics. Clinical validity was evaluated using known-groups methods to determine whether the OISA, OIDAS and OIQ-Composite scores were systematically related to illness severity. ME/CFS subjects were categorized into two groups according to their reported HUA over a 24-hour period, $<5$ HUA and $\geq 5$ HUA and employment status. Mean total OISA and OIDAS, means of the OISA and OIDAS components and mean OIQ-Composite scores were compared using ANOVA. 


\section{Results}

The characteristics of subjects are shown in Table 1. The demographics and baseline vitals of the ME/CFS subjects were similar to HCs. The majority of subjects were Caucasian (HC 92\% and ME/CFS $96 \%, p=0.54)$. The average HUA was 6.2 hours $(95 \% \mathrm{CI} \pm 1.09)$ in ME/CFS and 13.36 hours $(95 \%$ $\mathrm{CI} \pm 0.57)$ in HCs. The average duration of illness in ME/CFS was 14.67 years $(95 \% \mathrm{CI} \pm 4.21)$. Among HUA subgroups (Table 2), the duration of illness in ME/CFS subjects with $\geq 5$ HUA was 13.4 years $(95 \% \mathrm{CI} \pm 2.57)$ and 17.8 years $(95 \% \mathrm{CI} \pm 7.07)$ with $<5$ HUA $(p=0.41)$. The employment rate was $76 \%$ in $\mathrm{HCs}$ and $32 \%$ in ME/CFS, $p=0.001$. Furthermore, none of the $<5$ HUA ME/CFS subjects were employed compared to $44 \%$ of the $\geq 5$ HUA ME/CFS subjects $(p=0.03)$.

Orthostatic intolerance had a profound effect on all daily activities for ME/CFS subjects. All 6 upright activity items of the OIDAS were significantly different $(p<0.0001)$ between ME/CFS and HCs (Table 3). Interestingly, there were no differences in OIDAS scores between $<5$ HUA and $\geq 5$ HUA ME/CFS groups with both experiencing similar levels of interference in daily upright activities involving standing or walking. There were significant differences in the types of OI symptoms experienced by the $<5$ and $\geq 5$ HUA ME/CFS subjects (Table 4). Specifically, $<5$ HUA ME/CFS subjects had more problems with vision $(p=0.04)$, more weakness $(p=0.04)$ and more trouble concentrating $(p=0.04)$ compared to $\geq 5$ HUA ME/CFS subjects. There were no significant differences in dizziness, lightheadedness, feeling faint, or blackout, fatigue and head/neck discomfort.

OIQ is the combined score of OIDAS (possible 60 ) and OISA (possible 40) to equal a total score of possible 100. Total OIDAS and OISA scores between HCs and ME/CFS showed clear differences (Table 5); OIDAS: HC 5.08, MECFS 25.32, $p<0.001$; OISA: HC 6.72, MECFS 33.88, $p<0.001$ ). The total OIQ scores were significantly different between $<5$ HUA and $\geq 5$ HUA groups with the $<5$ HUA ME/CFS subjects having higher (worse) OIQ scores $(p=0.04)$.

Table 6 compares the study subjects based on OIQ scores and employment status. ME/CFS subjects that were employed had on average 8.62 HUA while ME/CFS subjects who were not working

Table 1

Characteristics of subjects

\begin{tabular}{lccc}
\hline & HC Total & ME/CFS Total & $P$-Value \\
\cline { 2 - 4 } Demographics $[ \pm \mathrm{CI}]$ & 25 & 25 & \\
\hline Age & $37.76[ \pm 5.95]$ & $40.92[ \pm 5.1]$ & 0.43 \\
Race $(\%)$ & & & \\
$\quad$ White & 92.0 & 96.0 & 0.56 \\
$\quad$ Other & 8.0 & 4.0 & 0.56 \\
HUA (Hour) & $13.36[ \pm 0.57]$ & $6.2[ \pm 1.09]$ & $<0.001$ \\
Duration of illness (Year) & - & $14.67[ \pm 4.21]$ & - \\
Employment (\%) (n) & $76(n=19)$ & $32(n=8)$ & $<0.001$ \\
Baseline vital (Supine position) & & & \\
$\quad$ SBP & $119.04[ \pm 5.93]$ & $117.44[ \pm 5.21]$ & 0.69 \\
DBP & $78.32[ \pm 3.08]$ & $76.88[ \pm 2.59]$ & 0.49 \\
$H R$ & $66.12[ \pm 4.12]$ & $68.75[ \pm 4.44]$ & 0.4 \\
\hline
\end{tabular}

Table 2

Characteristics of ME/CFS subjects based on HUA

\begin{tabular}{lccc}
\hline & $<5$ HUA & $\geq 5$ HUA & $P$-Value \\
\cline { 2 - 4 } Demographics $[ \pm \mathrm{CI}]$ & 7 & 18 & \\
\hline Age & $47.14[ \pm 5.45]$ & $38.5[ \pm 4.78]$ & 0.14 \\
Race $(\%)$ & 100 & 94.44 & 0.54 \\
$\quad$ White & 0 & 5.6 & 0.54 \\
$\quad$ Other & $2.86[ \pm 0.37]$ & $7.5[ \pm 0.83]$ & $<0.0001$ \\
HUA (Hours) & $17.83[ \pm 7.07]$ & $13.4[ \pm 2.57]$ & 0.41 \\
Duration of illness (Year) & $0(n=0)$ & $44.44(n=8)$ & 0.03 \\
Employment (\%) & & & \\
Baseline vital (Supine position) & $112.29[ \pm 3.85]$ & $119.4[ \pm 5.55]$ & 0.23 \\
$\quad S B P$ & $78.14[ \pm 1.66]$ & $76.39[ \pm 2.89]$ & 0.56 \\
$D B P$ & $69.53[ \pm 4.33]$ & $66.86[ \pm 4.81]$ & 0.61 \\
$H R$ & & & \\
\hline
\end{tabular}


Table 3

Mean scores $[ \pm \mathrm{CI}]$ for Orthostatic Intolerance Daily Activity Scale (OIDAS)

\begin{tabular}{|c|c|c|c|c|c|c|}
\hline & $\mathrm{HCs}$ & MECFS & $P$-value & $\mathrm{ME} / \mathrm{CFS}<5$ HUA & $\mathrm{ME} / \mathrm{CFS} \geq 5 \mathrm{HUA}$ & $P$-Value \\
\hline$n$ & 25 & 25 & & 7 & 18 & \\
\hline Standing a short time & $1[ \pm 0]$ & $4.28[ \pm 0.78]$ & $<0.0001$ & $4.57[ \pm 0.83]$ & $4.17[ \pm 0.31]$ & 0.66 \\
\hline Standing a long time & $1.72[ \pm 0.92]$ & $8.2[ \pm 0.77]$ & $<0.0001$ & $9[ \pm 0.83]$ & $7.89[ \pm 0.5]$ & 0.21 \\
\hline Walking a short time & $1[ \pm 0]$ & $4.32[ \pm 0.74]$ & $<0.0001$ & $4.29[ \pm 0.48]$ & $4.33[ \pm 0.83]$ & 0.96 \\
\hline Walking a long time & $1.36[ \pm 0.48]$ & $8.52[ \pm 0.9]$ & $<0.0001$ & $9.57[ \pm 0.61]$ & $8.11[ \pm 0.96]$ & 0.16 \\
\hline Average scores of items & $1.27[ \pm 0.29]$ & $6.33[ \pm 0.67]$ & $<0.0001$ & $6.13[ \pm 0.72]$ & $6.86[ \pm 0.48]$ & 0.35 \\
\hline Average scores of total OIDAS & $5.08[ \pm 1.19]$ & $25.32[ \pm 2.69]$ & $<0.0001$ & $27.43[ \pm 1.94]$ & $24.5[ \pm 2.92]$ & 0.35 \\
\hline
\end{tabular}

Table 4

Mean scores $[ \pm \mathrm{CI}]$ for Orthostatic Intolerance Symptom Assessment (OISA)

\begin{tabular}{|c|c|c|c|c|c|c|}
\hline & $\mathrm{HCs}$ & MECFS & $P$-value & $\mathrm{ME} / \mathrm{CFS}<5$ HUA & $\mathrm{ME} / \mathrm{CFS} \geq 5$ HUA & $P$-Value \\
\hline$\underline{n}$ & 25 & 25 & & 7 & 18 & \\
\hline $\begin{array}{l}\text { 1) Dizziness, lightheadedness, feeling } \\
\text { faint or blackout }\end{array}$ & $1.16[ \pm 0.21]$ & $5.8[ \pm 0.94]$ & $<0.0001$ & $7.14[ \pm 0.76]$ & $5.28[ \pm 0.94]$ & 0.08 \\
\hline 2) Problems with vision & $1.04[ \pm 0.07]$ & $3.84[ \pm 1.05]$ & $<0.0001$ & $5.57[ \pm 1.48]$ & $3.13 .17[ \pm 0.72]$ & 0.04 \\
\hline 3) Weakness & $1.12[ \pm 0.23]$ & $5.8[ \pm 1.13]$ & $<0.0001$ & $7.71[ \pm 0.86]$ & $5.06[ \pm 1.1]$ & 0.04 \\
\hline 4) Fatigue & $1.12[ \pm 0.17]$ & $7.08[ \pm 0.95]$ & $<0.0001$ & $8.48[ \pm 0.74]$ & $6.56[ \pm 0.97]$ & 0.22 \\
\hline 5) Trouble concentrating & $1.04[ \pm 0.07]$ & $6.28[ \pm 0.94]$ & $<0.0001$ & $7.86[ \pm 0.76]$ & $5.67[ \pm 0.9]$ & 0.04 \\
\hline 6) Head/Neck discomfort & $1.24[ \pm 0.28]$ & $5.08[ \pm 1.13]$ & $<0.0001$ & $6[ \pm 1.23]$ & $4.72[ \pm 1.09]$ & 0.33 \\
\hline Average scores of items & $1.12[ \pm 0.01]$ & $5.65[ \pm 1.31]$ & $<0.0001$ & $7.12[ \pm 1.71]$ & $5.07[ \pm 1.27]$ & 0.02 \\
\hline Average scores of total OISA & $6.72[ \pm 0.63]$ & $33.88[ \pm 1.5]$ & $<0.0001$ & $42.71[ \pm 2.18]$ & $30.44[ \pm 1.45]$ & 0.02 \\
\hline
\end{tabular}

Table 5

Mean OIQ scores $[ \pm \mathrm{CI}]$

\begin{tabular}{|c|c|c|c|c|c|c|}
\hline & $\mathrm{HCs}$ & MECFS & $P$-value & $\mathrm{ME} / \mathrm{CFS}<5$ HUA & $\mathrm{ME} / \mathrm{CFS} \geq 5 \mathrm{HUA}$ & $P$-Value \\
\hline$n$ & 25 & 25 & & 7 & 18 & \\
\hline Average scores of total OIDAS & $5.08[ \pm 1.19]$ & $25.32[ \pm 2.69]$ & $<0.0001$ & $27.43[ \pm 1.94]$ & $24.5[ \pm 2.92]$ & 0.35 \\
\hline Average scores of total OISA & $6.72[ \pm 0.28]$ & $33.88[ \pm 1.33]$ & $<0.0001$ & $42.71[ \pm 1.23]$ & $30.44[ \pm 1.09]$ & 0.02 \\
\hline OIQ composite & $11.8[ \pm 1.53]$ & $59.2[ \pm 6.64]$ & $<0.0001$ & $70.14[ \pm 6.28]$ & $54.94[ \pm 6.15]$ & 0.04 \\
\hline
\end{tabular}

Table 6

Mean scores $[ \pm \mathrm{CI}]$ for OIQ based on employment status

\begin{tabular}{|c|c|c|c|c|c|c|}
\hline & $\mathrm{HCs}$ & MECFS & $P$-value & ME/CFS Employed & ME/CFS Unemployed & $P$-Value \\
\hline$n$ & 25 & 25 & & 8 & 17 & \\
\hline Average scores of total OIDAS & $5.08[ \pm 1.19]$ & $25.32[ \pm 2.69]$ & $<0.0001$ & $22.13[ \pm 3.52]$ & $26.82[ \pm 2.06]$ & 0.35 \\
\hline Average scores of total OISA & $6.72[ \pm 0.63]$ & $33.88[ \pm 4.66]$ & $<0.0001$ & $27.25[ \pm 3.29]$ & $37[ \pm 4.79]$ & 0.02 \\
\hline OIQ composite & $11.8[ \pm 1.53]$ & $59.2[ \pm 6.64]$ & $<0.0001$ & $49.38[ \pm 5.8]$ & $63.82[ \pm 6.36]$ & 0.04 \\
\hline HUA (Hours) & $13.36[ \pm 0.57]$ & $6.2[ \pm 1.09]$ & $<0.0001$ & $8.62[ \pm 0.93]$ & $5.06[ \pm 0.78]$ & 0.001 \\
\hline
\end{tabular}

had on average 5.06 HUA ( $p=0.001)$. The OIQ scores were significantly higher for unemployed ME/CFS subjects (63.82) compared to employed ME/CFS subjects (49.38) $(p=0.04)$. The unemployed ME/CFS subjects reported significantly higher OISA scores than the employed group $(p=0.05)$, while there was no significant difference in total OIDAS scores (employed group: 22.13, unemployed group: $26.82, p=0.11$ ).

\section{Discussion}

Lightheadedness, headache, fatigue, weakness, heart palpitations, and exercise intolerance are some of the symptoms of orthostatic intolerance. Various physiological irregularities can underlie orthostatic symptoms including postural orthostatic tachycardia syndrome and orthostatic hypotension [10]. While questions still exist concerning the exact role of orthostatic intolerance in ME/CFS, increasing evidence shows substantial comorbidity [11]. Indeed, the Institute of Medicine report on clinical diagnostic criteria for ME/CFS lists orthostatic intolerance as one of the core diagnostic features of the illness [1]. Head-up tilt table testing and continuous heart rate monitoring are used in the research of OI, however, neither are modalities readily available to clinicians. Our goal was to use simple assessments to determine the 
impact of orthostatic symptoms and daily activities in ME/CFS patients to show the utility of HUA and OIQ for ME/CFS patients.

Clinical observation of ME/CFS patients at the Bateman Horne Center over the past 15 years indicates that patients who report more time in bed or reclining and less time with feet on the floor (e.g., sitting at a desk or table, standing, walking) were more severely ill. This observation was borne out by this study. ME/CFS subjects had significantly less HUA and significantly higher OIQ scores compared to HCs. This means that all upright postures - sitting with feet on the floor, standing, walking, running - exacerbate orthostatic intolerance and cause patients' orthostatic symptoms to flare. Patients report that can it take days for symptoms to subside following prolonged upright activity, and recovery requires them to lie down, elevate legs and feet, and rehydrate. Further, patients that have been sick for many years have learned to mitigate or prevent OI symptoms by managing energy expenditure (pacing), and especially, minimizing or avoiding exertion that keeps them in an upright position for longer than they know they can tolerate. This may help explain why ME/CFS subjects reported similar interference with daily activities due to OI on the OIDAS despite differences in HUA $(<5$ or $\geq 5$ ).

ME/CFS is debilitating and causes extreme limitations in a person's ability to accomplish even the most mundane of daily activities, including sitting upright at the dining table, standing to make a salad, taking a shower, driving a car, grocery shopping and cleaning the house. So, the $67 \%$ unemployment rate in our ME/CFS study subjects is not surprising. The data showed that ME/CFS subjects who were able to work had an average HUA of 8.62 a day in contrast to unemployed ME/CFS subjects who had an average HUA of 5 a day. Recall that the OIDAS score was not different between $<5$ and $\geq 5$ HUA ME/CFS subjects. Even though a person with ME/CFS maintains employment status, it should not be assumed that they can sustain normal work productivity without accommodations, normal physical function, or even the ability to engage in routine activities after work hours. If moderately ill ME/CFS patients spend all of their hours of upright activity at work, they often have nothing left for home, family, self-care and social activities. Therefore, clinicians should carefully consider each patient's orthostatic symptom severity and actual upright activity tolerance as it relates to performance in the workplace.
A recent paper described the importance of documenting medical disability in ME/CFS and the importance of documenting a reduction in the ability to carry out daily or work-related activities because of their disease [7]. The medical record documenting impaired physical function caused by ME/CFS is crucial for patients who need to claim Social Security disability due to the absence of ME/CFS within the Social Security List of Impairment [12]. There is a Social Security Regulation (SSR-14-1) that explains how impairments of ME/CFS constitute a determinable condition [13]. Both HUA and OIQ are simple assessments that can be used by the physician to document the impact of ME/CFS on physical function and daily activities. This documentation is not only useful for disability, but it is also essential for the effective diagnosis, treatment and ongoing management of ME/CFS patients [7]. ME/CFS is a multi-system illness, so simply assessing the presence of fatigue or OI is not enough to address the full clinical presentation and symptom severity. Clinicians must comprehensively assess all of the patient's major symptoms, including frequency, severity, and contribution to impaired physical function. This study focused on tools to recognize impaired function (HUA) and the presence of orthostatic intolerance; both are core clinical manifestations and possibly the most commonly overlooked aspects of ME/CFS illness. These tools can lead to better medical documentation, diagnosis and management as well as justification for engaging in more time-consuming objective measurements of OI such as the 10 minute Lean Test or Tilt Table testing.

In this study the relationship between self-reported hours of upright activity and the severity of orthostatic intolerance as assessed by a validated questionnaire was examined. We found that fewer hours of upright activity correlated with more severe orthostatic intolerance, which in turn increased the likelihood of being unemployed or underemployed. There are few studies demonstrating the assessment of symptoms and daily limitations of physical function in ME/CFS as described in this report. The ability to assess impairment and orthostatic symptom severity is meaningful and can be used as a tool for clinicians in the care of their ME/CFS patients. These results can also be used for educating patients about the importance of managing orthostatic intolerance in order to help improve daily function.

This study is limited by its relatively small sample size, enrollment of ME/CFS subjects from a single clinic, and the inclusion of only female subjects. The 
OHQ was developed and validated for orthostatic hypotension and the validity and reliability of this questionnaire for assessing orthostatic intolerance in ME/CFS is not known. The self-report of HUA is also a study limitation. However, the results of this small study showed stark differences between HCs and ME/CFS subjects on almost all measures, indicating that HUA is a valuable measure for ME/CFS and has inspired us to develop devices that objectively and passively measure HUA. Further study is required to validate these findings in a larger, more diverse population, including men, with consideration of stage/duration of illness and the presence of comorbid conditions.

\section{Acknowledgments}

This study was supported by the Chronic Fatigue Initiative at Hutchins Family Foundation.

\section{Conflict of interest}

None to report.

\section{References}

[1] IOM (Institute of Medicine). Beyond Myalgic Encephalomyelitis/Chronic Fatigue Syndrome: Redefining an Illness; The National Academies: Washington, DC, USA, 2015. doi:10.17226/19012

[2] The Voice of the Patient: Chronic Fatigue Syndrome and Myalgic Encephalomyelitis. Public Meeting: 4/25/2013; Report date: 9/2013. FDA (Food and Drug Administration).
[3] Stewart JM, Medow MS, Messer ZR, Baugham IL, Terilli $\mathrm{C}$, Ocon AJ. Postural neurocognitive and neuronal activated cerebral blood flow deficits in young chronic fatigue syndrome patients with postural tachycardia syndrome. Am J Physiol Heart Circ Physiol. 2012;302(5):H1185-94.

[4] Streeten DH, Anderson GH Jr. The role of delayed orthostatic hypotension in the pathogenesis of chronic fatigue. Clin Auton Res. 1998;8(2):119-24.

[5] Gerrity TR, Bates J, Bell DS, Chrousos G, Furst G, Hedrick T, Hurwitz B, Kula RW, Levine SM, Moore RC, Schondorf R. Chronic fatigue syndrome: what role does the autonomic nervous system play in the pathophysiology of this complex illness? Neuroimmunomodulation. 2002-2003;10(3):13441. Review.

[6] Low PA, Sandroni P, Joyner M, Shen WK. Postural tachycardia syndrome (POTS). J Cardiovasc Electrophysiol. 2009;20(3):352-8.

[7] Comerford BB, Podell R. Medically Documenting Disability in Myalgic Encephalomyelitis/Chronic Fatigue Syndrome (ME/CFS) Cases. Front Pediatr. 2019;7:231.

[8] Fukuda K, Straus SE, Hickie I, Sharpe MC, Dobbins JG, Komaroff A. The chronic fatigue syndrome: a comprehensive approach to its definition and study. International Chronic Fatigue Syndrome Study Group. Ann Intern Med. 1994;121(12):953-9.

[9] Carruthers BM, Jain AK, De Meirleir KL, Peterson DL, Klimas NG, Lerner AM, Sherkey JA. Myalgic encephalomyelitis/chronic fatigue syndrome: clinical working case definition, diagnostic and treatment protocols. Journal of Chronic Fatigue Syndrome. 2003;11(1):7-115.

[10] Kaufmann H, Malamut R, Norcliffe-Kaufmann L, Rosa K, Freeman R. The Orthostatic Hypotension Questionnaire (OHQ): validation of a novel symptom assessment scale. Clin Auton Res. 2012;22(2):79-90.

[11] Stewart JM. Common syndromes of orthostatic intolerance. Pediatrics. 2013;131(5):968-80.

[12] Social Security. Listing of Impairments - Adult Listings (Part A) [Internet]. Disability Evaluation Under Social Security. Available from: https://www.ssa.gov/disability/ professionals/bluebook/AdultListings.htm

[13] SSR 14-1p: Titles II and XVI: Evaluating Cases Involving Chronic Fatigue Syndrome (CFS). Available from: https:// www.ssa.gov/OP_Home/rulings/di/01/SSR2014-01-di-01. html. 